\title{
MathematicalModel For Finding ACPM Of MTBF Projection In Plasma Vasopressin And Response To Treatment In Primary Nocturnal Enuresis
}

\author{
S. Lakshmi * and M. Senbagavalli ** \\ * Principal, Government of Arts and Science College, Peravurani, Thanjavur, TamilNadu. \\ ** Research Scholar, Department of Mathematics, Kunthavai Naacchiyaar Govt. Arts College for Women \\ (Autonomous), Thanjavur, TamilNadu.
}

\begin{abstract}
In this paper, we apply the results on both the model AMSAA Crow projection model and Reliability growth potential effects of vasopressin to examine the relation between nocturnal vasopressin release and response to treatment with the vasopressin analogue 1-desamino-8-D-Arginine Vasopressin (DDAVP) in children with primary mono symptomatic nocturnal enuresis. In this application the ability to respond to DDAVP is related to endogenous AVP production and is influenced by neuronal patterning in early infancy. The result concludes that ACPM of MTBF projection is estimated of the system failure intensity. $\hat{\rho}_{\mathrm{GP}}$ is the growth potential of Mean time between failure of AVP release according to the result in the estimated growth potential failure intensity, the estimate of the system MTBF growth potential. Thus the ACPM Projection for the system failure intensity, based on $\widehat{\beta}$, the corresponding MTBF Projection value is nearly equal to the Projected system failure intensity based on $\bar{\beta}_{\mathrm{m}}$, the corresponding MTBF Projection values.
\end{abstract}

Keywords:- Vasopressin, DDVAP, Nocturnal Enuresis, AMSAA Crow projection model, Reliability growth potential.

\section{INTRODUCTION}

The aetiology of nocturnal enuresis have shown that a number of children have a failure of the normal circadian rhythm of vasopressin, resulting in large volumes of urine being produced at night[15,17]. This has led to the therapeutic rationale of using the vasopressin analogue 1-desamino-8- D-arginine vasopressin (DDAVP) as a treatment for nocturnal enuresis, with a number of placebo controlled studies showing a significant response to treatment. The response to medication varies between publications owing to the different definitions of outcome, usually defined by frequency of dry nights while on medication, rather than the cure rate off medication. Our study investigated this issue by examining both the relation between nocturnal AVP plasma concentrations and response to DDAVP, together with an analysis of which physiological factors might influence the response. The attainment of nocturnal continence appears in part to depend on the development of a circadian rise in AVP resulting in reduced urine production at night. In children with low concentrations of AVP there is a direct relation between the mean plasma AVP concentration and response. The lower the AVP the less likely the child is to respond to DDAVP. The confounding factor is the influence of previous breast feeding on response to DDAVP. Breast feeding is known to enhance early neuronal maturation and visual acuity, which may be important in the entrainment of normal circadian rhythmicity and in the establishment of normal neuronal pathways[13]. It is proposed that children with enuresis and low concentrations, particularly those breast fed as infants, have established their normal neuronal pathways, but there is a failure to produce the normal circadian release of AVP at night. However, this may be mimicked by giving exogenous AVP [14].

\section{NOTATION}

$\begin{array}{ll}\lambda & \text {-Scale Parameter } \\ \beta & \text {-Shape Parameter } \\ \mathrm{t} & \text { - Test time } \\ \mathrm{T} & \text {-Total test time } \\ \text { MTBF } & \text { - Mean time between failures } \\ \lambda_{\mathrm{i}} & \text {-The i-th successive failure time } \\ \mathrm{N} & \text { - Total number of failures } \\ \mathrm{N}_{\mathrm{A}} & \text {-Number of A mode failures } \\ \mathrm{N}_{\mathrm{B}} & \text {-Number of B mode failures } \\ \lambda_{\mathrm{A}} & \text {-Type A modes failure intensity } \\ \lambda_{\mathrm{B}} & \text {-Type B modes failure intensity }\end{array}$


$\widehat{\beta}-$ ACPM Projected system failure intensity

$\bar{\beta}_{\mathrm{m}} \quad$ - Projected system failure intensity

$\hat{\rho}_{c}(T) \quad$-ACPM projection for the system failure intensity

$\hat{\rho}_{\mathrm{GP}} \quad-$ Growth potential Mean time between failure.

\subsection{AMSAA-Crow Projection Model (ACPM).}

\section{MATHEMATICAL MODEL}

This model considers the case where all corrective actions to surfaced B-modes are implemented at the end of the current test phase, prior to commencing a follow-on test phase. Thus all fixes are delayed, making it a test-find-test strategy. The current test phase will be referred to as Phase I and the follow-on test phase as Phase II.

The ACPM and associated parameter estimation procedure was developed to assess the reliability impact of a group of delayed fixes. In particular, the model and estimation procedure allows assessment of what the system failure intensity will be at the start of Phase II after implementation of the delayed fixes[3,6 ]. Denoting this failure intensity by $\mathrm{r}(\mathrm{T})$, where $\mathrm{T}$ denotes the duration of Phase I, the ACPM assessment of $\mathrm{r}(\mathrm{T})$ is based on: (1) the A and B-mode failure data generated during Phase I test duration, T; and (2) assessments of the FEFs for the B-modes surfaced during Phase I. Since the assessments of the FEFs are often largely based on engineering judgment, the resulting assessment, $\widehat{r}(T)$, of the system failure intensity after corrective action implementations is called a reliability projection (as opposed to a demonstrated assessment, which would be based solely on test data)[7].

The ACPM and estimation procedure was motivated by the desire to replace the widely used "adjustment procedure". The adjustment procedure assesses $\mathrm{r}(\mathrm{T})$ based on reducing the number of failures, $\mathrm{N}_{\mathrm{i}}$, due to B-mode I during Phase I to $\left(1-d_{i}^{*}\right) N_{i}$, where $d_{i}{ }^{*}$ is the assessment of the realized FEF for mode $i$, denoted by $d_{i}$. Note $\left(1-\mathrm{d}_{\mathrm{i}}^{*}\right) \mathrm{N}_{\mathrm{i}}$ is an assessment of the expected number of failures due to B-mode I that would occur in a follow-on test of the same duration as Phase I[8]. The adjustment procedure assesses $r(T)$ by $\hat{r}_{\text {adj }}(T)$, where $\hat{r}_{\text {adj }}(T)=\frac{N_{A}}{T}+$ $\frac{\sum_{i \in o b s}\left(1-\mathrm{d}_{\mathrm{i}}^{*}\right) N_{i}}{T}$

In this equation, obs is the index set for all the B-modes that occur during Test Phase I.

The ACPM assesses the value of the system failure intensity, $\mathrm{r}(\mathrm{T})$, after implementation of the Phase I delayed fixes. This assessment is taken to be an estimate of the expected value of $\mathrm{r}(\mathrm{T})$, i.e., an estimate of $\rho_{C}(T)$ $=E(r(T))$. Crow proposed an approximation for the ACPM approach which can be expressed as

$$
\rho_{c}(T)=\lambda_{A}+\sum_{i=1}^{K}\left(1-d_{i}\right) \lambda_{i}+\mu_{d} \cdot h_{c}(T)
$$

Where the first two terms are equivalent to $\hat{\mathrm{r}}_{\text {adj }}(\mathrm{T})$ above [1,9].

In this expression, $\lambda_{A}$ denotes the assumed constant A-mode failure rate, $\lambda_{\mathrm{i}}$ denotes the initial B-mode failure rate for mode $i, \mu_{\mathrm{d}}$ is the assumed common mean of all the FEFs when considered as random variables for the K B-modes, $d_{i}$ denotes the realized value of the achieved FEF for mode $i$ if mode $i$ is surfaced. The function $h_{c}(T)$ represents the rate of occurrence of new B-modes at the end of the test Phase.

Based on an empirical study, Crow states that the number of distinct B-modes surfaced over a test period $[0, t]$ can often be approximated by a power function of the form

$$
\mu_{c}(t)=\lambda t^{\beta} \quad \text { for } \lambda, \beta>0
$$

This function is interpreted as the expected number of distinct B-modes surfaced during the test interval $[0, t]$. More specifically, it assumes the number of distinct B-modes occurring over $[0, t]$ is governed by a NHPP with $\mu_{c}(t)$ as the mean value function [4].

Thus $\mathrm{h}_{\mathrm{c}}(\mathrm{t})=\frac{d \mu_{c}(t)}{d t}=\lambda \beta \mathrm{t}^{\beta-1}$

represents the expected rate at which new B-modes are occurring at test time $t$.

For estimating , the first term in the expression is constant over $[0, T]$ and is estimated by

$\hat{\lambda}_{\mathrm{A}}=\frac{\mathrm{N}_{\mathrm{A}}}{T}$

Where $\mathrm{N}_{\mathrm{A}}$ is the number of A-mode failure over [0,T].

Based on the assumption that all fixes are delayed until Phase I is complete, the failure rate for B-mode $\mathrm{i}$ remains constant over $[0, T]$ and is estimated by

$$
\hat{\lambda}_{\mathrm{i}}=\frac{\mathrm{N}_{\mathrm{i}}}{T} \quad(\mathrm{i}=1,2 \ldots \ldots \ldots \mathrm{K})
$$

Where $\mathrm{N}_{\mathrm{i}}$ denotes the number of failures during [0,T] attributable to B-mode i, [5]. Note

$$
\mathrm{E}\left(\hat{\lambda}_{\mathrm{i}}\right)=\frac{\mathrm{E}(\mathrm{Ni})}{\mathrm{T}}=\frac{\lambda_{i} T}{T}=\lambda_{\mathrm{i}}
$$


The next step to complete the assessment of the expected system failure intensity after incorporation of delayed fixes is to address the rate of occurrence of new B-modes at $T, h_{c}(t)=\lambda \beta t^{\beta-1}$.

The data required to estimate $\lambda$ and $\beta$ are:

*The number of distinct $\mathrm{B}$-modes, $\mathrm{m}$, that occur during $[0, \mathrm{~T}]$

*The B-mode first occurrence times $0<\mathrm{t}_{1} \leq \mathrm{t}_{2} \leq \ldots \leq \mathrm{t}_{\mathrm{m}} \leq \mathrm{T}$.

The maximum likelihood estimates of $\lambda$ and $\beta$, denoted by $\hat{\lambda}$ and $\hat{\beta}$ respectively, satisfy the following equations:

$$
\widehat{\beta}=\frac{\hat{\mathrm{\lambda}} T^{\widehat{\beta}}}{\sum_{\mathrm{i}=1}^{\mathrm{m}} \operatorname{In}\left(\frac{\mathrm{T}}{\mathrm{t}_{\mathrm{i}}}\right)} \mathrm{m}
$$

Solving for $\hat{\lambda}$ in the above, the estimate for $h_{c}(T)$ can be in terms of $m$ and $\hat{\beta}$ as follows:

$$
\hat{h}(\mathrm{~T})=\hat{\lambda} \widehat{\beta} \mathrm{T}^{\hat{\beta}-1}=\left(\frac{m}{T^{\hat{\beta}}}\right) \hat{\beta} \mathrm{T}^{\hat{\beta}-1}=\frac{\mathrm{m} \hat{\beta}}{T}
$$

Crow notes that conditioned on the observed number of distinct B-modes, $\mathrm{m}$, i.e., $\mathrm{M}(\mathrm{T})=\mathrm{m}$,

the estimator [10],

is unbiased estimator of $\beta$, i.e.,

$$
\bar{\beta}_{\mathrm{m}}=\left(\frac{m-1}{m}\right) \hat{\beta} \quad \mathrm{m} \geq 2
$$

$$
\mathrm{E}\left(\bar{\beta}_{\mathrm{m}}\right)=\beta
$$

Thus estimating $\mathrm{h}_{\mathrm{c}}(\mathrm{T})=\lambda \mathrm{t}^{\beta-1}$ by using $\bar{\beta}_{\mathrm{m}}$ leads to estimate

$$
\bar{h}_{\mathrm{c}}(\mathrm{T})=\frac{m \bar{\beta}_{m}}{T}
$$

To complete the assessment of the system failure intensity, it is necessary to assess the ACPM expected system failure intensity $\rho_{\mathrm{c}}(\mathrm{T}),[1]$,

Based on $\widehat{\beta}$ and since $\mathrm{N}_{\mathrm{i}}=0$ for $\mathrm{i} \notin o b s$

$$
\rho_{\mathrm{c}}(\mathrm{T})=\lambda_{\mathrm{A}}+\sum_{\mathrm{i}=1}^{\mathrm{K}}\left(1-\mathrm{d}_{\mathrm{i}}\right) \lambda_{\mathrm{i}}+\mu_{\mathrm{d}} \cdot \mathrm{h}_{\mathrm{c}}(\mathrm{T})
$$

$$
\hat{\rho}_{c}(\mathrm{~T})=\frac{1}{T}\left\{\mathrm{~N}_{\mathrm{A}}+\sum_{\mathrm{i} \in \mathrm{obs}}\left(1-\mathrm{d}_{\mathrm{i}}^{*}\right) \mathrm{N}_{\mathrm{i}}+\widehat{\beta} \sum_{\mathrm{i} \in \mathrm{obs}} \mathrm{d}_{\mathrm{i}}^{*}\right\}
$$

For $\rho_{\mathrm{c}}(\mathrm{T})$ based on $\bar{\beta}_{\mathrm{m}}($ provided $\mathrm{m} \geq 2)$ :

$$
\bar{\rho}_{\mathrm{c}}(\mathrm{T})=\frac{1}{T}\left\{\mathrm{~N}_{\mathrm{A}}+\sum_{\mathrm{i} \in \mathrm{obs}}\left(1-\mathrm{d}_{\mathrm{i}}^{*}\right) \mathrm{N}_{\mathrm{i}}+\bar{\beta} \mathrm{m} \sum_{\mathrm{i} \in \mathrm{obs}} \mathrm{d}_{\mathrm{i}}^{*}\right\}
$$

\subsection{Reliability Growth Potential}

The expected system failure intensity after incorporation of the delayed fixes decreases to a limiting value as $\mathrm{T} \rightarrow \infty$. This value is termed the failure intensity growth potential and is denoted by $\rho_{\mathrm{GP}}$. It is given by

$$
\rho_{\mathrm{GP}}=\lim _{n \rightarrow \infty} \rho_{\mathrm{c}}(\mathrm{T})=\lambda_{\mathrm{A}}+\sum_{\mathrm{i}=1}^{\mathrm{K}}\left(1-\mathrm{d}_{\mathrm{i}}\right) \lambda_{i}
$$

Its reciprocal is referred to as the MTBF growth potential, which represents a theoretical upper limit on the system MTBF. This limit corresponds to the MTBF that would result if all B-mode failures were surfaced and corrected with specified FEFs. Note that $\rho_{\mathrm{GP}}$

is estimated by

$$
\hat{\rho}_{\mathrm{GP}}=\frac{1}{T}\left(N_{A}+\sum_{i \in o b s}\left(1-d_{i}^{*}\right) N_{i}\right)
$$

If the reciprocal $\left(\hat{\rho}_{G P}\right)^{-1}$ lies below the goal MTBF, then this may indicate that achieving the goal is high risk.

\section{APPLICATION}

\subsection{Effects of AVP Release on primary nocturnal enuresis}

The pattern of AVP release changes with an increasing variability associated with higher plasma concentrations. This may reflect increasing central release as part of establishing the normal circadian rhythm or, as we postulate, it could be related to bladder filling and AVP release stimulated by bladder contraction. Watanabe has examined the relation between sleep state and nocturnal arousal caused by a full bladder and describes, within a subgroup, uninhibited forceful bladder contractions leading to urination without arousal[18]. Other studies have suggested that bladder distension might result in AVP release[16]. Therefore, the bladder may try to regulate urine production by initiating AVP release as it fills. Although during the day this eventually results in the desire to micturate, at night, it is necessary to be aroused from sleep first before the bladder empties. Only by specific bladder pressure studies would it be possible to show that high pressure bladder contractions during sleep would result in pulses of AVP as seen in these children. The attainment of nocturnal continence may first be associated with the development of the circadian rhythm of AVP and then "fine tuned" by the bladder stimulating AVP release once a certain volume is reached. The combination of 
DDAVP and anti cholinergic medication may be useful in patients with nocturnal enuresis and has already been used and shown to be beneficial [2].Our study was designed to investigate specifically children with primary mono symptomatic enuresis. The inclusion criteria were strict to reduce confounding variables to a minimum, so that children with daytime wetting and children with developmental problems were not included.

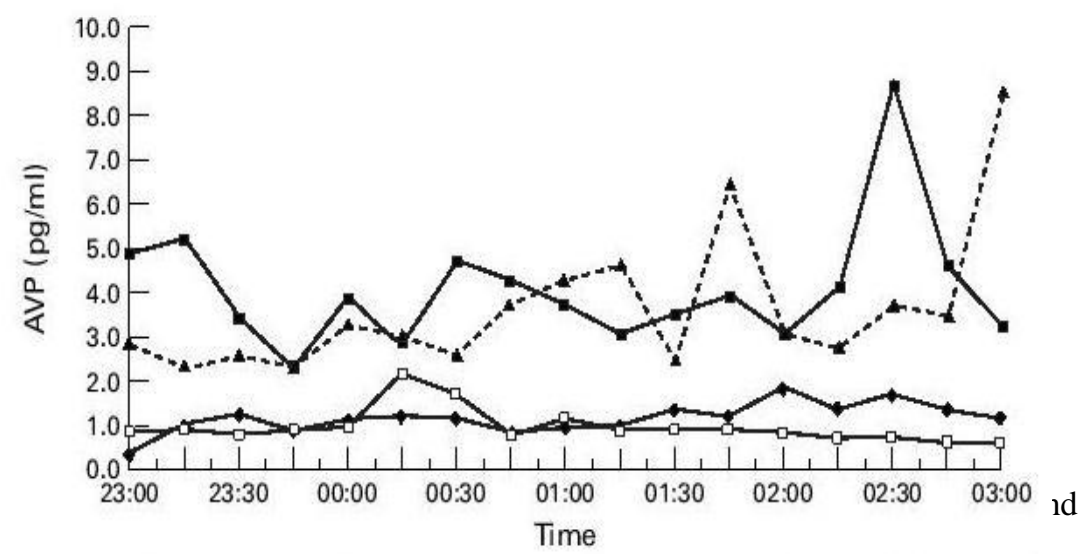

\subsection{Effects of relation between AVP Release on primary nocturnal enuresis}

The study confirms the importance of AVP release at night in the establishment of nocturnal continence. In particular we have shown the importance of the hypothalamic axis and how this may be influenced adversely by perinatal events and advantageously by breast feeding. There appear to be at least two possible aetiologies of nocturnal enuresis. One is associated with a low concentration of AVP, where there may be a failure to produce the circadian rise in AVP but normal neural pathways exist. We have suggested that the bladder itself may stimulate AVP release in an attempt to finely tune the control of night time urine production. The second is associated with high AVP concentrations and severe nocturnal enuresis. The aetiology must be speculative but our data suggest that AVP receptor failure might be involved in night time continence. Jarvelin and Fergusson have suggested that primary and secondary enuresis are aspects of the same problem, the primary form being regarded as a delay in maturation of the physiological mechanisms that lead to continence[12,11]. There might be a progressive developmental process in the establishment of nocturnal continence. This involves the development of a circadian rise in AVP resulting in reduction in urine volume. In conjunction with this is the production of AVP in relation to bladder contraction and the ability to awake to a full bladder. Although functional bladder volume might be a contributory factor, it may not be part of the developmental process involved primarily in nocturnal continence.

\section{MATHEMATICAL RESULTS}

The Application part is the stimulation with

$\mathrm{N}$ is total number of increasing A mode and decreasing B mode of AVP release,

$\mathrm{N}_{\mathrm{A}}$ is the number of increasing $\mathrm{A}$ mode,

$\mathrm{N}_{\mathrm{B}}$ is the number of decreasing $\mathrm{B}$ mode,

$\lambda_{\mathrm{A}}$ is the type A mode increasing AVP,

$\lambda_{\mathrm{B}}$ is the type B mode decreasing AVP,

$\bar{\beta}_{\mathrm{m}}$ is the Projected system of increasing AVP release,

$\hat{\rho}_{c}(T)$ is the Projection for the system increasing AVP release,

$\hat{\rho}_{\mathrm{GP}}$ is the growth potential of Mean time between failure of AVP release according to the

result in the estimated growth potential failure intensity is $\hat{\mathrm{r}}_{\mathrm{adj}}(\mathrm{T})=\hat{\rho}_{\mathrm{GP}}=0.0455$,

The estimate of the system MTBF growth potential is $\rho_{\mathrm{GP}}{ }^{-1}=21.9648$.

Thus the ACPM Projection for the system failure intensity, based on $\widehat{\beta}$, is $\hat{\rho}_{c}(\mathrm{~T})=0.0531$,

the corresponding MTBF Projection is $\left\{\hat{\rho}_{c}\right\}^{-1}=18.83$,

the Projected system failure intensity based on $\bar{\beta}_{\mathrm{m}}$, is $\bar{\rho}_{\mathrm{c}}(\mathrm{T})=0.0525$,

the corresponding MTBF Projection is $\left\{\bar{\rho}_{c}\right\}^{-1}=19.04$.

\section{CONCLUSION}

Here, we apply the results on both the model AMSAA Crow projection model and Reliability growth potential effects of vasopressin to examine the relation between nocturnal vasopressin release and response to treatment with the vasopressin analogue 1-desamino-8-D-Arginine Vasopressin (DDAVP) in children with 
primary mono symptomatic nocturnal enuresis. The result concludes that ACPM Projection for the system failure intensity, based on $\widehat{\beta}$, the corresponding MTBF Projection value is nearly 18.83 equal to the Projected system failure intensity based on $\bar{\beta}_{\mathrm{m}}$, the corresponding MTBF Projection values 19.04.

\section{REFERENCES}

[1] AMSAA.” MIL-HDBK-189: Reliability Growth Management.” APG, Feb 1981.Broemm, Ellner, and Woodworth. AMSAA Reliability Growth Guide.APG: AMSAA TR-652,Sep 2000.

[2] Caione PL, Giorgio PL, Passerine-Glazel G, Desmopressin (DDAVP) and oxybutynin in nocturnal enuresis: results of a multi centred trial. International Children's Continence Society monograph series No 1.Tunbridge Wells: Wells Medical Ltd, 1995.

[3] Crow, Larry H. “ AMSAA Discrete Reliability Growth Model.” APG: Methodology office Note 1-83, 1983.

[4] Crow, Larry H. An Improved Methodology for Reliability Growth Projections. APG: AMSAA Technical Report TR-357, Jun 1982.

[5] Crow, Larry H. Reliability Analysis for Complex Repairable Systems.APG: AMSAA Technical Report TR-138, 1974.

[6] Eller, Paul M., and Bruce Trapnell. AMSAA Reliability Growth Data Study.APG: AMSAA Interim Note IN-R-184, 1990.

[7] Eller, Paul M., and Brian Hall. "AMSAA Maturity Projection Model Based on Stein Estimation ." Technical Report TR-751, APG, 2004.

[8] Eller, Paul M., and Brian Hall. Planning Model Based on Projection Methodology (PM2). APG: AMSAA Technical Report TR-2006-09, Mar 2006.

[9] Eller, Paul M., and Lindalce C. wald. "AMSAA Maturity Projection Model.” 1995 Proceedings of Annual Reliability and Maintainability Symposium. Jan 1995.

[10] Eller, Paul M., and Robert Mioduski. Operating Characteristic Analysis for Reliability Growth Programs. APG: AMSAA Technical Report TR-524, Aug 1992.

[11] Ferguson DM, Horwood CT, Shanon FT. Secondary enuresis in a birth cohort of New Zealand children. Paed Perinat Epidemiol1990;4:53-63.

[12] Jarvelin, . Etiological and precipitating factors for childhood enuresis. Acta Paediatr Scand1991;80:3619.

[13] Kalo BB, Bella $\mathrm{H}$. Enuresis: prevalence and associated factors among primary school children in Saudi Arabia. Acta Paediatr Scand1996;85:1217-22.

[14] Makrides M, Neumann M, Simmer K, Pater J, Gibson R.Are long-chain polyunsaturated fatty acids essential nutrients in infancy? Lancet 1995;345:1463-8.

[15] Norgaard JP, Pedersen EB, Djurhuus JC. Diurnal antidiuretic hormone levels in enuretics. J Urol1985;134:1029-31.

[16] Ohne T,.Theincrease in C-fos expression in vasopressin and oxytocin immunoreactive neurons in paraventricular and supraoptic nucleus of the hypothalamus following urinary retention. J Kyoto Pref Univ Med1995;104:393-403.

[17] Rittig S, Knudsen UB, Norgaard JP, Pedersen EB, Djurhuus JC. Abnormal diurnal rhythm of plasma vasopressin and urinary output in patients with enuresis. Am J Physiol1989;256:F664-71.

[18] Watanabe H. Sleep problems in children with nocturnal enuresis. Scand J UrolNephrol Suppl 1995; 173:55-8. 